\title{
Reduction of optimum light power with Heisenberg-limited photon-counting noise in interferometric gravitational-wave detectors
}

\author{
Constantin Brif* \\ LIGO Project, California Institute of Technology, Pasadena, CA 91125
}

\begin{abstract}
We study how the behavior of quantum noise, presenting the fundamental limit on the sensitivity of interferometric gravitational-wave detectors, depends on properties of input states of light. We analyze the situation with specially prepared nonclassical input states which reduce the photoncounting noise to the Heisenberg limit. This results in a great reduction of the optimum light power needed to achieve the standard quantum limit, compared to the usual configuration.
\end{abstract}

04.80.Nn, 42.50.Dv

Since the pioneering work by Caves [1,2], it is well understood that two sources of quantum noise - the photon-counting noise and the radiation-pressure noiseconstitute the fundamental limitation on the sensitivity of an interferometric gravitational-wave detector. These limitations will be of potential importance in longbaseline interferometric detectors which are currently under construction (the LIGO project [3,4] in the United States and the French-Italian VIRGO project [5] in Europe are the largest ones). For example, the photoncounting shot noise will dominate at the gravitationalwave frequencies above $1 \mathrm{kHz}$ in the VIRGO detector and above $200 \mathrm{~Hz}$ in the initial LIGO detector. With a further reduction of the thermal noise, planned in the advanced LIGO interferometer, the role of the shot noise will be even more important.

For a coherent laser beam of light power $P$, the shot noise associated with photon-counting statistics scales as $P^{-1 / 2}$ and the radiation-pressure noise scales as $P^{1 / 2}$. The contributions of these two sources of noise will be equal for some optimum value $P_{\mathrm{opt}}$ of the light power. Provided that classical sources of noise (such as thermal and seismic) are sufficiently suppressed, the interferometer with the optimum light power will work at the socalled standard quantum limit (SQL). A simple quantum calculation, based on the use of the Heisenberg uncertainty principle, gives the SQL for the measurement of the relative shift $z=z_{2}-z_{1}$ in the positions of two end mirrors:

$$
(\Delta z)_{\mathrm{SQL}}=\sqrt{2 \hbar \tau / m}
$$

where $m$ is the mass of each end mirror and $\tau$ is the measurement time. For modern long-baseline interferometers (like LIGO and VIRGO), Fabry-Perot cavities are used in the arms; so $\tau$ is actually the cavity storage time, $\tau=L \mathcal{F} / \pi c$, where $L$ is the cavity length, $\mathcal{F}$ is the finesse, and $c$ is the velocity of light. The optimum power, for which the SQL is achieved, is

$$
P_{\mathrm{opt}}=\frac{m L^{2}}{\omega \tau^{4}},
$$

where $\omega$ is the light angular frequency. For the initial LIGO configuration, the mirror mass is $m \simeq 11 \mathrm{~kg}$, the cavity length is $L \simeq 4 \mathrm{~km}$, the finesse is $\mathcal{F} \simeq 200$, and the wavelength of the Nd:YAG laser is $\lambda \simeq 1.064 \mu \mathrm{m}$ $\left(\omega \simeq 1.77 \times 10^{15} \mathrm{~Hz}\right)$. This gives the cavity storage time $\tau \simeq 8.5 \times 10^{-4} \mathrm{~s}$ and an effective number of bounces $b=\tau c / 2 L \simeq 32$. The corresponding optimum laser power is $P_{\mathrm{opt}} \simeq 191 \mathrm{~kW}$ and the SQL of the position shift measurement is $(\Delta z)_{\mathrm{SQL}} \simeq 1.24 \times 10^{-19} \mathrm{~m}$. Achieving this SQL will make possible to measure gravitational waves with amplitudes $h$ greater than $\sim 3 \times 10^{-23}$.

Presently, the available laser light power is insufficient for achieving the SQL (for example, in the initial LIGO configuration the input laser power is $6 \mathrm{~W}$ and the power recycling gain is about 30 ). Therefore, in advanced LIGO configurations, it is planned [- 4 to reduce the shot noise by using more powerful lasers, in conjunction with the power-recycling technique [6, ,7]. However, for very high laser power, one encounters serious technical problems related to nonuniform heating of the cavity mirrors caused by absorption of even a small portion of circulating light. The resulting thermal aberrations can seriously deteriorate the performance of the interferometer [7]. Therefore, it will be very interesting to study possibilities for achieving the SQL with low light power.

The gravitational-wave detection community is quite familiar with the intriguing idea by Caves [2] to reduce the photon-counting noise by squeezing the vacuum fluctuations at the unused input port. During the last decade, other interesting ideas has been developed in the field of theoretical quantum optics, based on the use of nonclassical photon states for the quantum noise reduction in idealized optical interferometers [8 13]. The main

*E-mail: cbrif@ligo.caltech.edu 
theoretical motivation of all those papers was to show the possibility of beating the shot-noise limit and achieving the fundamental Heisenberg limit for the photon-counting noise in an ideal interferometric measurement.

The aim of the present work is to show that the optimum light power needed for the SQL operation of an interferometric gravitational-wave detector with movable mirrors can be greatly reduced by the use of nonclassical states of light with the Heisenberg-limited photoncounting noise. This result means that Heisenberglimited interferometry is not only interesting for a demonstration of the fundamental uncertainty principle, but can be also important for the experimental detection of gravitational waves.

Let us consider a long-baseline Michelson interferometer whose arms are equipped with high-finesse FabryPerot cavities, with end mirrors serving as free test masses. In the quantum description, two modes of the light field enter the interferometer through the two input ports of a 50-50 beam splitter. After being mixed in the beam splitter, the light modes spent time $\tau$ in the Fabry-Perot cavities, and then leave the interferometer (through the same beam splitter, but in the opposite direction). The photons leaving the interferometer in the output modes are counted by two photodetectors. A gravitational wave incident on the interferometer will cause a relative shift $z=z_{2}-z_{1}$ in the positions of two end mirrors, which results in the phase shift $\phi=(\omega \tau / L) z$ between the two arms.

The performance of such an interferometer can be analyzed in the Heisenberg picture, using a nice grouptheoretic description proposed by Yurke et al. [8]. Using the boson annihilation operators $a_{1}$ and $a_{2}$ of the two input modes, one constructs the operators

$$
\begin{aligned}
& J_{x}=\left(a_{1}^{\dagger} a_{2}+a_{2}^{\dagger} a_{1}\right) / 2, \\
& J_{y}=-\mathrm{i}\left(a_{1}^{\dagger} a_{2}-a_{2}^{\dagger} a_{1}\right) / 2, \\
& J_{z}=\left(a_{1}^{\dagger} a_{1}-a_{2}^{\dagger} a_{2}\right) / 2 .
\end{aligned}
$$

These operators form the two-boson realization of the $\mathrm{su}(2)$ Lie algebra, $\left[J_{k}, J_{l}\right]=\mathrm{i} \epsilon_{k l m} J_{m}$. The Casimir operator is a constant, $\mathbf{J}^{2}=j(j+1)$, for any unitary irreducible representation of the $\mathrm{SU}(2)$ group; so the representations are labeled by a single index $j$ that takes the values $j=0,1 / 2,1,3 / 2, \ldots$. The representation Hilbert space $\mathcal{H}_{\mid}$is spanned by the complete orthonormal basis $|j, m\rangle(m=j, j-1, \ldots,-j)$. Using Eq. (3), one finds

$$
\mathbf{J}^{2}=\frac{1}{2} N\left(\frac{1}{2} N+1\right), \quad N=a_{1}^{\dagger} a_{1}+a_{2}^{\dagger} a_{2},
$$

where $N$ is the total number of photons entering the interferometer. We see that $N$ is an $\mathrm{SU}(2)$ invariant; if the input state of the two-mode light field belongs to $\mathcal{H}_{1}$, then $N=2 j$.

The actions of the interferometer elements on the column-vector $\mathbf{J}=\left(J_{x}, J_{y}, J_{z}\right)^{T}$ can be represented as rotations in the 3 -dimensional space [8]. The first mixing in the beam splitter produces a rotation around the $y$ axis by $-\pi / 2$, with the transformation matrix $\mathrm{R}_{y}(-\pi / 2)$. The second mixing corresponds to the opposite rotation, with the transformation matrix $\mathrm{R}_{y}(\pi / 2)$. The relative phase shift produces a rotation around the $z$ axis by $\phi$, with the transformation matrix $\mathrm{R}_{z}(\phi)$. The overall transformation performed on $\mathbf{J}$ is the rotation by $\phi$ around the $x$ axis,

$$
\mathrm{R}_{x}(\phi)=\mathrm{R}_{y}(\pi / 2) \mathrm{R}_{z}(\phi) \mathrm{R}_{y}(-\pi / 2) .
$$

The information on the phase shift $\phi$ is inferred from the photon statistics of the output beams. Usually, one measures the difference between the number of photons in the two output modes,

$$
q_{\mathrm{out}}=2 J_{z \mathrm{out}}=2\left[(\sin \phi) J_{y}+(\cos \phi) J_{z}\right]
$$

If we assume that there are no losses in the interferometer and the classical sources of noise are well suppressed, then the uncertainty in the relative position shift $z$ of the end mirrors is due to two factors [1]:2]. The first one is the photon-counting noise. Indeed, since there are quantum fluctuations in $q_{\text {out }}$, a phase shift is detectable only if it induces a change in $\left\langle q_{\text {out }}\right\rangle$ which is larger than the uncertainty $\Delta q_{\text {out }}$. Consequently, the uncertainty in the phase shift due to the photon-counting noise is

$$
(\Delta \phi)_{\mathrm{pc}}^{2}=\frac{\left(\Delta q_{\mathrm{out}}\right)^{2}}{\left(\partial\left\langle q_{\mathrm{out}}\right\rangle / \partial \phi\right)^{2}} .
$$

If the detection is made on a dark fringe $(\phi=\pi / 2$ in the unperturbed state), then the contribution of the photoncounting noise is

$$
(\Delta z)_{\mathrm{pc}}^{2}=A_{\mathrm{pc}} \frac{\left(\Delta J_{y}\right)^{2}}{\left\langle J_{z}\right\rangle^{2}}, \quad A_{\mathrm{pc}}=\left(\frac{L}{\omega \tau}\right)^{2} .
$$

The second source of noise is due to quantum fluctuations in the radiation pressure. The difference between the momenta transferred by light to the end mirrors, $\mathcal{P}=p_{2}-p_{1}$, is easily found to be $\mathcal{P}=(2 \hbar \omega \tau / L) J_{x}$. The relative shift in the positions of the end mirrors, due to the transferred momenta, is $(\tau / m) \mathcal{P}$. Therefore, the contribution of the radiation-pressure noise is

$$
(\Delta z)_{\mathrm{rp}}^{2}=A_{\mathrm{rp}}\left(2 \Delta J_{x}\right)^{2}, \quad A_{\mathrm{rp}}=\left(\frac{\hbar \omega \tau^{2}}{m L}\right)^{2} .
$$

Consider the standard case when the coherent laser beam of amplitude $\alpha$ enters the interferometer's one input port, while the vacuum enters the other. This input state $\mid$ in $\rangle=|\alpha\rangle_{1}|0\rangle_{2}$ (where $|0\rangle$ is the vacuum and $|\alpha\rangle=\exp \left(\alpha a^{\dagger}-\alpha^{*} a\right)|0\rangle$ is the coherent state $)$, satisfies

$$
\begin{gathered}
\left\langle J_{x}\right\rangle=\left\langle J_{y}\right\rangle=0, \quad\left\langle J_{x}^{2}\right\rangle=\left\langle J_{y}^{2}\right\rangle=|\alpha|^{2} / 4, \\
\left\langle J_{z}\right\rangle=|\alpha|^{2} / 2, \quad\langle N\rangle \equiv \bar{N}=|\alpha|^{2} .
\end{gathered}
$$


Using these results, one finds

$$
(\Delta z)^{2}=(\Delta z)_{\mathrm{pc}}^{2}+(\Delta z)_{\mathrm{rp}}^{2}=A_{\mathrm{pc}} \bar{N}^{-1}+A_{\mathrm{rp}} \bar{N} .
$$

Optimizing $(\Delta z)^{2}$ as a function of $\bar{N}$, one obtains

$$
\bar{N}_{\text {opt }}=\frac{m L^{2}}{\hbar \omega^{2} \tau^{3}},
$$

and $P_{\text {opt }}=\hbar \omega \bar{N}_{\text {opt }} / \tau$ is given by Eq. (2), while the optimum value of $\Delta z$ is the SQL of Eq. (11).

The characteristic noise behavior of Eq. (10) is sometimes explained by the Poissonian photon statistics of the coherent state (i.e., by the fact that $\Delta N_{1}=\left\langle N_{1}\right\rangle^{1 / 2}$, with $\left.N_{1}=a_{1}^{\dagger} a_{1}\right)$. However, it is not difficult to see that this explanation is principally wrong. A simple calculation shows that if instead of the coherent state $|\alpha\rangle$ at the first input port we will use an arbitrary state (pure or mixture) of the single-mode light field, the same result (10) will hold. This will be true, regardless of the statistical properties of the photon state at the first input port, as long as the vacuum enters the second input port.

Caves [2] proposed to reduce the optimum power needed to achieve the SQL by using the squeezed vacuum in the second input port. If the carrier mode entering the first input port is in the coherent state $|\alpha\rangle$, the two-mode input state is given by $\mid$ in $\rangle=|\alpha\rangle_{1}|\xi\rangle_{2}$, where $|\xi\rangle=\exp \left(\frac{1}{2} \xi a^{\dagger 2}-\frac{1}{2} \xi^{*} a^{2}\right)|0\rangle$ (with $\left.\xi=r \mathrm{e}^{\mathrm{i} \theta}\right)$ is the squeezed vacuum state. If one takes $\theta=0$ and real $\alpha$, the input state satisfies

$$
\begin{gathered}
\left\langle J_{x}\right\rangle=\left\langle J_{y}\right\rangle=0, \quad\left\langle J_{x, y}^{2}\right\rangle=\left(\alpha^{2} \mathrm{e}^{ \pm 2 r}+\sinh ^{2} r\right) / 4, \\
\left\langle J_{z}\right\rangle=\left(\alpha^{2}-\sinh ^{2} r\right) / 2, \quad \bar{N}=\alpha^{2}+\sinh ^{2} r .
\end{gathered}
$$

In the usual situation, $\alpha^{2} \gg \sinh ^{2} r$, so one derives

$$
(\Delta z)^{2} \simeq A_{\mathrm{pc}} \mathrm{e}^{-2 r} \bar{N}^{-1}+A_{\mathrm{rp}} \mathrm{e}^{2 r} \bar{N}
$$

The use of the squeezed vacuum reduces the photoncounting noise at the expense of the radiation-pressure noise. This results in the reduced optimum light power:

$$
P_{\text {opt }}(r) \simeq P_{\text {opt }}(r=0) \mathrm{e}^{-2 r},
$$

while the optimum value of $\Delta z$ remains the SQL of Eq. (11). However, it is erroneous to think that the reduction of the optimum light power is the merit of the squeezed vacuum alone; actually, the state of the carrier mode is important as well. While the state in the carrier mode was mixed with the phase-insensitive vacuum, the behavior of the quantum noise was determined by the mean number of carrier photons only. However, when mixing the carrier mode with a phase-sensitive state (e.g., with the squeezed vacuum), the precise matching between the quantum states of the two modes is important. For example, if the state of the carrier mode satisfies $\left\langle a_{1}^{\dagger 2}+a_{1}^{2}\right\rangle=0$ and $\left\langle N_{1}\right\rangle \gg \sinh ^{2} r$, then we obtain

$$
(\Delta z)^{2} \simeq\left(A_{\mathrm{pc}} \bar{N}^{-1}+A_{\mathrm{rp}} \bar{N}\right) \cosh 2 r
$$

This results in the same optimum power (2) as for the normal vacuum case, but the sensitivity deteriorates: $(\Delta z)_{\mathrm{opt}}^{2} \simeq(\Delta z)_{\mathrm{SQL}}^{2} \cosh 2 r$. For example, this will be the case for the carrier mode in the coherent state $|\alpha\rangle$ with $\arg \alpha=\pi / 4$ or in any phase-insensitive state (a state is called phase-insensitive, if its density matrix is diagonal in the Fock basis; examples are the Fock states themselves or the thermal state). On the other hand, if the carrier mode in an arbitrary state is mixed with a phase-insensitive state (or, more generally, with any state satisfying $\left.\left\langle a_{2}^{2}\right\rangle=0\right)$, then

$$
(\Delta z)^{2}=\left(2 \bar{N}_{1} \bar{N}_{2}+\bar{N}_{1}+\bar{N}_{2}\right)\left[A_{\mathrm{pc}}\left(\bar{N}_{1}-\bar{N}_{2}\right)^{-2}+A_{\mathrm{rp}}\right] .
$$

Here, we used notation $\bar{N}_{k}=\left\langle a_{k}^{\dagger} a_{k}\right\rangle, k=1,2$. Clearly, the quantum noise cannot be reduced here, compared to the vacuum case; in particular, for $\bar{N}_{1} \gg \bar{N}_{2}$, the optimum power remains as in Eq. (2), but the sensitivity deteriorates: $(\Delta z)_{\mathrm{opt}}^{2} \simeq(\Delta z)_{\mathrm{SQL}}^{2}\left(1+2 \bar{N}_{2}\right)$.

From the above arguments, one understands that the reduction of the optimum power can be achieved with a proper phase matching between the two input modes. In this relation, it is interesting to consider input states which lead to the Heisenberg-limited photon-counting noise 813 . It is well known that the shot-noise limit $(\Delta \phi)_{\mathrm{pc}}=N^{-1 / 2}$, achieved with the vacuum at the second input port, is not a fundamental one. Using the uncertainty relation $\left(\Delta J_{x}\right)\left(\Delta J_{y}\right) \geq \frac{1}{2}\left|\left\langle J_{z}\right\rangle\right|$, one obtains $(\Delta \phi)_{\mathrm{pc}} \geq\left(2 \Delta J_{x}\right)^{-1}$. Since for any input state $\mid$ in $\rangle \in \mathcal{H}_{j}$ the relation $\left(\Delta J_{x}\right)^{2} \leq \frac{1}{2} j(j+1)$ holds, one finds the Heisenberg limit

$$
(\Delta \phi)_{\mathrm{pc}} \geq[2 j(j+1)]^{-1 / 2} .
$$

Consequently, for large photon numbers $(\bar{N}=2 j \gg 1)$, the photon-counting noise $(\Delta z)_{\mathrm{pc}}$ scales as $1 / P$.

It can be shown that the shot-noise limit can be surpassed with the so-called intelligent (minimumuncertainty) states 11, 12. (The use of intelligent states for achieving the Heisenberg limit in spectroscopy was discussed in [14].) The $J_{x}-J_{y}$ intelligent states, by their definition, equalize the uncertainty relation: $\left(\Delta J_{x}\right)\left(\Delta J_{y}\right)=\frac{1}{2}\left|\left\langle J_{z}\right\rangle\right|$. These states are determined by the eigenvalue equation

$$
\left(\eta J_{x}-\mathrm{i} J_{y}\right)|\lambda, \eta\rangle=\lambda|\lambda, \eta\rangle
$$

The spectrum is discrete: $\lambda=\mathrm{i} m_{0} \sqrt{1-\eta^{2}}$, where $m_{0}=j, j-1, \ldots,-j$, and $\eta$ is a real parameter given by $|\eta|=\Delta J_{y} / \Delta J_{x}$. Recently, a method for experimental generation of the $\mathrm{SU}(2)$ intelligent states was proposed in 15. 
If the two-mode light field entering the interferometer is prepared in the $J_{x}-J_{y}$ intelligent state, the quantum noise takes the form

$$
(\Delta z)^{2}=A_{\mathrm{pc}}\left(2 \Delta J_{x}\right)^{-2}+A_{\mathrm{rp}}\left(2 \Delta J_{x}\right)^{2} .
$$

For $|\eta|<1$, the intelligent states are squeezed in $J_{y}$ and anti-squeezed in $J_{x}$, thereby reducing the photoncounting noise below the shot-noise limit, on account of increasing contribution of the radiation-pressure noise. For $\eta \rightarrow 0$, one obtains [16]

$$
\left(2 \Delta J_{x}\right)^{2}=2\left|\left\langle J_{z}\right\rangle / \eta\right| \simeq 2\left(j^{2}-m_{0}^{2}+j\right),
$$

and the Heisenberg limit for the photon-counting noise is achieved when $m_{0}=0$. Then, for large photon numbers $(\bar{N}=2 j \gg 1)$, we obtain

$$
(\Delta z)^{2} \simeq 2 A_{\mathrm{pc}} \bar{N}^{-2}+\frac{1}{2} A_{\mathrm{rp}} \bar{N}^{2}
$$

Optimizing $(\Delta z)^{2}$ as a function of $\bar{N}$, we find $(\Delta z)_{\mathrm{opt}} \simeq$ $(\Delta z)_{\mathrm{SQL}}$, but the optimum light power needed to achieve the SQL is dramatically reduced:

$$
\bar{N}_{\mathrm{opt}}=\left(\frac{2 m L^{2}}{\hbar \omega^{2} \tau^{3}}\right)^{1 / 2}, \quad P_{\mathrm{opt}}=\left(\frac{2 \hbar m L^{2}}{\tau^{5}}\right)^{1 / 2}
$$

Using the parameters for the initial LIGO, we find the values $\bar{N}_{\text {opt }} \simeq 4.3 \times 10^{10}$ and $P_{\text {opt }} \simeq 9 \mu \mathrm{W}$. Compare these values with those obtained in the standard configuration (with the vacuum at the second port): $\bar{N}_{\text {opt }} \simeq 9.2 \times 10^{20}$ and $P_{\text {opt }} \simeq 191 \mathrm{~kW}$. We see that the use of the intelligent states with the Heisenberg-limited photon-counting noise can in principle reduce the optimum light power by a factor $\sim 2 \times 10^{10}$.

There were proposals to achieve the Heisenberg limit for the photon-counting noise by driving the interferometer with two Fock states containing equal numbers of photons [9,13. (The use of Fock states was also proposed for Heisenberg-limited interferometry with matter waves [17 and for Heisenberg-limited spectroscopy with degenerate Bose-Einstein gases [18].) The corresponding input state is $\mid$ in $\rangle=|n\rangle_{1}|n\rangle_{2}=|j, 0\rangle$ with $j=n=\bar{N} / 2$. Clearly, this input state cannot be used when one measures the photon difference $q_{\text {out }}$ at the output. However, as was shown in Ref. [13], the Heisenberg-limited photoncounting noise is achieved by measuring the squared difference $S=q_{\text {out }}^{2}=4 J_{z \text { out }}^{2}$ at the output. (We do not discuss here technical problems involved in this kind of measurement.) In this case, the uncertainty in the phase shift due to the photon-counting noise is

$$
(\Delta \phi)_{\mathrm{pc}}^{2}=\frac{(\Delta S)^{2}}{(\partial\langle S\rangle / \partial \phi)^{2}}=\frac{\tan ^{2} \phi}{8}+\frac{2-\tan ^{2} \phi}{4 j(j+1)} .
$$

For $\phi=0$ (this corresponds to a dark fringe for the measurement of $S$ ), the Heisenberg limit is achieved:
$(\Delta \phi)_{\mathrm{pc}}=[2 j(j+1)]^{-1 / 2}$. Of course, this improvement is on account of the corresponding increase in the radiationpressure noise, because $\left(\Delta J_{x}\right)^{2}=\frac{1}{2} j(j+1)$ takes its maximum value. Therefore, for large photon numbers, we obtain $(\Delta z)_{\mathrm{pc}} \simeq\left(2 A_{\mathrm{pc}}\right)^{1 / 2} / \bar{N}$ and $(\Delta z)_{\mathrm{rp}} \simeq\left(A_{\mathrm{rp}} / 2\right)^{1 / 2} \bar{N}$ recovering the result of Eq. (19). In fact, this quantum noise behavior and the corresponding reduction of the optimum power are characteristic for input states with the Heisenberg-limited photon-counting noise.

It should be emphasized that above results are valid for a lossless interferometer, while mirrors of realistic detectors (for example, LIGO) do have losses. The Heisenberglimited photon-counting noise can be achieved only if the losses are sufficiently small. Let $\Gamma$ be the dimensionless coefficient of losses, defined by $\bar{N}_{\text {out }}=\bar{N} \mathrm{e}^{-\Gamma} \simeq \bar{N}(1-\Gamma)$. In the case of input Fock states, a simple analysis shows 19 that the Heisenberg-limited photon-counting noise $(\Delta \phi)_{\mathrm{pc}} \sim \bar{N}^{-1}$ can be obtained only for $\bar{N} \Gamma<1 / 2$. Sure, the value $\Gamma \sim 10^{-11}$ is impossible to achieve with the present technology. The problem of losses is of great practical importance but nevertheless it does not cross out the principal value of the idea to reduce the optimum light power by using nonclassical input states. For example, one can imagine a realization of this idea with a prototype interferometer which should have smaller $m$ and $L$ and larger $\tau$.

In conclusion, we analyzed the behavior of quantum noise, which limits the sensitivity of interferometric gravitational-wave detectors, for various input states of the light field. We found that by using nonclassical input states exhibiting the Heisenberg-limited photoncounting noise, the optimum light power needed to achieve the SQL can be significantly reduced, compared to the usual configuration. Of course, a practical realization of Heisenberg-limited interferometry will depend on future theoretical and experimental progress in the methods for production of stable and sufficiently powerful sources of nonclassical light and on technical solutions for the reduction of losses and classical sources of noise.

The author thanks Kip S. Thorne and John Preskill for stimulating discussions and Ady Mann and Albert Lazzarini for valuable comments on the manuscript. Financial support from the Lester Deutsch Fund is gratefully acknowledged. This work was supported in part by the Institute of Theoretical Physics at the Department of Physics at the Technion, and the author is grateful to the Institute for hospitality during his visit to the Technion. The LIGO Project is supported by the National Science Foundation under the cooperative agreement PHY9210038 . 
[1] C.M. Caves, Phys. Rev. Lett. 45 (1980) 75.

[2] C.M. Caves, Phys. Rev. D 23 (1981) 1693.

[3] A. Abramovici et al., Science 256 (1992) 325.

[4] J.K. Blackburn, in: Mathematics of Gravitation Part II, Gravitational Wave Detection (Banach Center Publications, Warsaw, 1997), p. 95.

[5] B. Caron et al., Nucl. Phys. B (Proc. Suppl.) 54B (1997) 167; Class. Quantum Grav. 14 (1997) 1461.

[6] R.W.P. Drever, in: Gravitational Radiation, ed. N. Deruelle and T. Piran (North-Holland, Amsterdam, 1983), p. 321 .

[7] P. Hello, in: Progress in Optics, Vol. 38, ed. E. Wolf (North-Holland, Amsterdam, 1998), p. 85.

[8] B. Yurke, S.L. McCall and J.R. Klauder, Phys. Rev. A 33 (1986) 4033.

[9] M.J. Holland and K. Burnett, Phys. Rev. Lett. 71 (1993) 1355.

[10] B.C. Sanders and G.J. Milburn, Phys. Rev. Lett. 75
(1995) 2944.

[11] M. Hillery and L. Mlodinow, Phys. Rev. A 48 (1993) 1548.

[12] C. Brif and A. Mann, Phys. Rev. A 54 (1996) 4505.

[13] T. Kim, O. Pfister, M.J. Holland, J. Noh and J.L. Hall, Phys. Rev. A 57 (1998) 4004.

[14] D.J. Wineland, J.J. Bollinger, W.M. Itano, F.L. Moore and D.J. Heinzen, Phys. Rev. A 46 (1992) R6797;

D.J. Wineland, J.J. Bollinger, W.M. Itano and D.J. Heinzen, Phys. Rev. A 50 (1994) 67;

G.S. Agarwal and R.R. Puri, Phys. Rev. A 49 (1994) 4968.

[15] A. Luis and J. Peřina, Phys. Rev. A 53 (1996) 1886.

[16] C. Brif, Int. J. Theor. Phys. 36 (1997) 1651.

[17] J.P. Dowling, Phys. Rev. A 57 (1998) 4736.

[18] P. Bouyer and M.A. Kasevich, Phys. Rev. A 56 (1997) R1083.

[19] J. Preskill, private communication. 